\title{
Comprehensive screening of alternative lengthening of telomeres phenotype and loss of ATRX expression in sarcomas
} Jau-Yu Liau ${ }^{1,2}$, Jen-Chieh Lee ${ }^{1,2}$, Jia-Huei Tsai ${ }^{1,2}$, Ching-Yao Yang ${ }^{3}$, Tsung-Lin Liu ${ }^{1,2}$,
Zhi-Long Ke ${ }^{1,2}$, Hung-Han Hsu ${ }^{1,2}$ and Yung-Ming Jeng

${ }^{1}$ Department of Pathology, National Taiwan University Hospital, College of Medicine, National Taiwan University, Taipei, Taiwan; ${ }^{2}$ Graduate Institute of Pathology, College of Medicine, National Taiwan

University, Taipei, Taiwan and ${ }^{3}$ Department of Surgery, National Taiwan University Hospital, College of

Medicine, National Taiwan University, Taipei, Taiwan

According to cytogenetic aberrations, sarcomas can be categorized as complex or simple karyotype tumors. Alternative lengthening of telomeres is a telomere-maintenance mechanism common in sarcomas. Recently, this mechanism was found to be associated with loss of either a-thalassemia/mental retardation syndrome X-linked (ATRX) or death domain-associated (DAXX) protein. We previously reported that alternative lengthening of telomeres and loss of ATRX expression were common in leiomyosarcoma, angiosarcoma, pleomorphic liposarcoma, and dedifferentiated liposarcoma. In the present study, we screened an additional 245 sarcomas of other types to determine the prevalence of alternative lengthening of telomeres, loss of ATRX/DAXX expression, and their relationship. Undifferentiated pleomorphic sarcomas were frequently alternative lengthening of telomeres positive (65\%) and loss of ATRX was seen in approximately half of the alternative lengthening of telomeres-positive tumors. Nineteen of 25 myxofibrosarcomas were alternative lengthening of telomerespositive, but only one was ATRX deficient. Three of 15 radiation-associated sarcomas were alternative lengthening of telomeres positive, but none of them was ATRX deficient. Alternative lengthening of telomeres and/or loss of ATRX were uncommon in malignant peripheral nerve sheath tumors, gastrointestinal stromal tumors, and embryonal rhabdomyosarcomas. By contrast, none of the $\mathbf{7 1}$ gene fusion-associated sarcomas was ATRX deficient or alternative lengthening of telomeres positive. All tumors exhibited preserved DAXX expression. Combining our previous studies and this study, a total of 384 sarcomas with complex karyotypes were examined, 83 of which were ATRX deficient (22\%). By telomere-specific fluorescence in situ hybridization, $45 \%(138 / 308)$ were alternative lengthening of telomeres positive, $55 \%(76 / 138)$ of which were ATRX deficient. Loss of ATRX was highly associated with alternative lengthening of telomeres $(P<0.001)$. We conclude that alternative lengthening of telomeres is a frequent telomere-maintenance mechanism in cytogenetically complex sarcomas. Loss of ATRX is highly associated with this feature.

Modern Pathology (2015) 28, 1545-1554; doi:10.1038/modpathol.2015.114; published online 2 October 2015

Soft tissue sarcomas are a heterogeneous group of tumors with diverse differentiations, clinical presentations, histomorphologies, molecular pathogeneses, and patient prognoses. According to cytogenetic aberrations, sarcomas are broadly categorized as: (1) sarcomas with complex karyotypic abnormalities that frequently have numerical

Correspondence: Professor Y-M Jeng, MD, PhD, Department of Pathology, National Taiwan University Hospital, No 7, Chung-Shan South Road, Taipei 10051, Taiwan.

E-mail: mrna0912@yahoo.com.tw

Received 20 June 2015; revised 12 August 2015; accepted 17 August 2015; published online 2 October 2015 changes, non-recurrent translocations, and deletions; and (2) sarcomas with simple karyotypes that typically involve fusion or mutation of specific genes. ${ }^{1}$ Histologically, tumors with complex karyotypes, such as leiomyosarcoma, pleomorphic liposarcoma, and conventional osteosarcoma, are characterized by nuclear pleomorphism and the presence of atypical mitotic figures. They frequently harbor TP53 mutations, but no other consistent genetic alteration has been identified. They are more common in older patients. By contrast, tumors with simple karyotypes, such as synovial sarcoma, myxoid liposarcoma, and Ewing sarcoma, typically exhibit nuclear uniformity, lack atypical mitotic 
figures, and are more common in young adults. The specific gene fusions or mutations occur in high frequencies and are highly valuable for diagnosis.

Telomeres, the repetitive hexameric TTAGGG sequences at the ends of each chromosome, are critical in maintaining chromosomal stability. ${ }^{2}$ Telomere length must be maintained for the immortalization of malignant cells. Two main telomeremaintenance mechanisms have been proposed: telomerase activation and the alternative lengthening of telomeres. ${ }^{2}$ Approximately $10-15 \%$ of cancers, notably gliomas and sarcomas, utilize alternative lengthening of telomeres as the telomeremaintenance mechanism. The alternative lengthening of telomeres-positive cells are characterized by marked telomere length heterogeneity and the presence of alternative lengthening of telomeresassociated promyelocytic leukemia bodies. ${ }^{3,4}$ Previous studies have revealed that alternative lengthening of telomeres is commonly used by some sarcomas with complex cytogenetics, such as undifferentiated pleomorphic sarcomas (malignant fibrous histiocytomas), leiomyosarcomas, and osteosarcomas. ${ }^{5-7}$ Montgomery et al. have suggested that alternative lengthening of telomeres is a feature of sarcomas with complex cytogenetics, but is rare in translocation-associated sarcomas. $^{8}$ Alternative lengthening of telomeres has been shown to be an unfavorable prognostic factor in several types of sarcomas. ${ }^{6,9-12}$

Until recently, the underlying mechanism of alternative lengthening of telomeres remains elusive. Heaphy et al. discovered that alternative lengthening of telomeres status was perfectly correlated with the loss of expression of either $\alpha$-thalassemia/mental retardation syndrome X-linked (ATRX) or death domain-associated (DAXX) protein in pancreatic neuroendocrine tumors. ${ }^{13}$ ATRX and DAXX form a dimer that acts as a histone chaperone to deposit histone variant H3.3 to GC-rich regions of the genome, including the telomeres, and plays important roles in maintaining telomere stability. ${ }^{14-16}$ It is hypothesized that dysfunction of the dimer leads to telomere instability, increased telomere homologous recombination, and ultimately, alternative lengthening of telomeres. Subsequent studies have reported frequent ATRX loss (but not DAXX loss) in astrocytoma, leiomyosarcoma, dedifferentiated liposarcoma and other tumor types, and the loss of ATRX has been highly correlated with the alternative

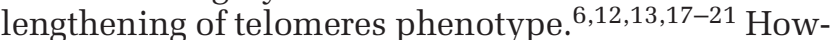
ever, knockdown of ATRX or DAXX has not led to alternative lengthening of telomeres, and patients with germline ATRX mutations do not appear to be cancer prone. ${ }^{18,22}$ In addition, although loss of either ATRX or DAXX is perfectly associated with alternative lengthening of telomeres in pancreatic neuroendocrine tumors, the situation is more complex in sarcomas. In our previous studies, only approximately half of alternative lengthening of telomeres- positive leiomyosarcomas and pleomorphic liposarcomas were ATRX deficient, ${ }^{6,12}$ but alternative lengthening of telomeres was highly correlated with loss of ATRX in angiosarcomas and dedifferentiated liposarcomas. ${ }^{12,23}$ Thus, current information indicates that alternative lengthening of telomeres is a manifestation of global telomere dysfunction exploiting dysregulated homologous recombination to elongate the telomeres. Loss of ATRX/DAXX is one of the mechanisms of alternative lengthening of telomeres, but other genetic changes must also be present concurrently for the alternative lengthening of telomeres phenotype to fully develop.

Although many sarcoma types have been evaluated for the prevalence of alternative lengthening of telomeres, the correlation of alternative lengthening of telomeres with loss of ATRX/DAXX expression remains largely unknown. We previously studied this telomere-maintenance mechanism and ATRX/DAXX expression in leiomyosarcomas, angiosarcomas, and liposarcomas. ${ }^{6,12,23}$ The aim of the present study was to systematically screen other sarcoma types to determine the frequency of alternative lengthening of telomeres, ATRX/DAXX expression, and their relationship.

\section{Materials and methods}

\section{Tumor Samples}

Sarcomas of various types (Tables 1 and 2) were retrieved from the archives of the Department of Pathology, National Taiwan University Hospital. Histological and immunohistochemical sections were reviewed to confirm the diagnoses. Tumors were classified to sarcomas with simple or complex genotypes based on the current knowledge. Cytogenetic analyses for individual tumors were not performed. This study was approved by the Research Ethics Committee of National Taiwan University Hospital, and the specimens were anonymous and analyzed in a blind manner.

\section{Immunohistochemistry and Telomere-Specific Fluorescence In Situ Hybridization (FISH)}

Immunohistochemistry was performed using ATRX (1:500, Sigma Aldrich, St Louis, MO, USA) and DAXX (1:500, Sigma Aldrich) antibodies, as previously described. ${ }^{6}$ For telomere-specific FISH, a fluorescein isothiocyanate-labeled peptide nucleic acid probe (Panagene, Daejeon, Korea) was used. ${ }^{6}$ The optimal protease $\mathrm{K}$ digestion time for each tumor was individually adjusted. The typical digestion times for each tumor type are as follows: 180 to $360 \mathrm{~s}$ for undifferentiated pleomorphic sarcomas, myxofibrosarcomas, malignant peripheral nerve sheath tumors, solitary fibrous tumors, alveolar soft part sarcomas, extraskeletal myxoid chondrosarcomas, and gastrointestinal stromal tumors; 
Table 1 ATRX immunohistochemistry, alternative lengthening of telomeres, and their correlations in sarcomas with complex cytogenetics

\begin{tabular}{|c|c|c|c|c|c|c|}
\hline \multirow{2}{*}{ Sarcomas with complex cytogenetics } & \multirow{2}{*}{$\mathrm{N}$} & \multirow{2}{*}{ ATRX loss } & \multicolumn{2}{|c|}{$\begin{array}{c}\text { Alternative lengthening } \\
\text { of telomeres (+) }\end{array}$} & \multicolumn{2}{|c|}{$\begin{array}{c}\text { Alternative lengthening } \\
\text { of telomeres }(-)\end{array}$} \\
\hline & & & $\mathrm{N}(\%)$ & ATRX loss & $\mathrm{N}(\%)$ & ATRX loss \\
\hline Undifferentiated pleomorphic sarcoma & 35 & $12 / 35(34 \%)$ & $22 / 34(65 \%)$ & $12 / 22(55 \%)$ & $12 / 34(35 \%)$ & $0 / 12(0 \%)$ \\
\hline Myxofibrosarcoma & 27 & $1 / 27(4 \%)$ & $19 / 25(76 \%)$ & $1 / 19(5 \%)$ & $6 / 25(24 \%)$ & $0 / 6(0 \%)$ \\
\hline Osteosarcoma & 18 & $4 / 18(22 \%)$ & N/A & N/A & N/A & N/A \\
\hline Malignant peripheral nerve sheath tumor & 17 & $1 / 17(6 \%)$ & $3 / 14(21 \%)$ & $1 / 3(33 \%)$ & $11 / 14(79 \%)$ & $0 / 11(0 \%)$ \\
\hline Embryonal rhabdomyosarcoma & 9 & $1 / 9(11 \%)$ & $1 / 8(13 \%)$ & $1 / 1(100 \%)$ & $7 / 8(88 \%)$ & $0 / 7(0 \%)$ \\
\hline Radiation-associated sarcoma & 20 & $0 / 20(0 \%)$ & $3 / 15(20 \%)$ & $0 / 3(0 \%)$ & $12 / 15(80 \%)$ & $0 / 12(0 \%)$ \\
\hline Chondrosarcoma & 15 & $0 / 15(0 \%)$ & N/A & N/A & N/A & N/A \\
\hline Total & 141 & $19 / 141(13 \%)$ & $48 / 96(50 \%)$ & $15 / 48(31 \%)$ & $48 / 96(50 \%)$ & $0 / 48(0 \%)$ \\
\hline \multicolumn{7}{|l|}{ Our previous studies ${ }^{\mathrm{a}}$} \\
\hline Leiomyosarcoma & 92 & $30 / 92(33 \%)$ & $51 / 86(59 \%)$ & $28 / 51(55 \%)$ & $35 / 86(41 \%)$ & $2 / 35(6 \%)$ \\
\hline Pleomorphic liposarcoma & 11 & $5 / 11(45 \%)$ & $8 / 10(80 \%)$ & $5 / 8(63 \%)$ & $2 / 10(20 \%)$ & $0 / 2(0 \%)$ \\
\hline Angiosarcoma & 88 & $16 / 88(18 \%)$ & $17 / 70(24 \%)$ & $15 / 17(88 \%)^{b}$ & $53 / 70(76 \%)$ & $0 / 53(0 \%)$ \\
\hline Dedifferentiated liposarcoma & 52 & $13 / 52(25 \%)$ & $14 / 46(30 \%)$ & $13 / 14(93 \%)^{\mathrm{c}}$ & $32 / 46(70 \%)$ & $0 / 32(0 \%)$ \\
\hline Total & 384 & $83 / 384(22 \%)$ & $138 / 308(45 \%)$ & $76 / 138(55 \%)$ & $170 / 308(55 \%)$ & $2 / 170(1 \%)$ \\
\hline
\end{tabular}

Abbreviation: N/A, non-available.

${ }^{a}$ References of our previous studies: leiomyosarcoma: Liau et al.; ${ }^{6}$ pleomorphic liposarcoma and dedifferentiated liposarcoma: Lee et al.; ${ }^{12}$ angiosarcoma: Liau et al..$^{23}$

${ }^{\mathrm{b}}$ Two alternative lengthening of telomeres (+) angiosarcomas were proficient for both ATRX and DAXX. Two ATRX-deficient angiosarcomas exhibited focal DAXX loss.

${ }^{\mathrm{c}}$ The only ATRX-proficient alternative lengthening of telomeres (+) dedifferentiated liposarcoma was DAXX deficient.

Table 2 ATRX immunohistochemistry, alternative lengthening of telomeres, and their correlations in fusion-associated sarcomas, gastrointestinal stromal tumors, and epithelioid sarcomas

\begin{tabular}{|c|c|c|c|c|c|c|}
\hline \multirow{2}{*}{ Sarcomas with fusion genes } & \multirow{2}{*}{$\mathrm{N}$} & \multirow{2}{*}{ ATRX loss } & \multicolumn{2}{|c|}{$\begin{array}{c}\text { Alternative lengthening } \\
\text { of telomeres (+) }\end{array}$} & \multicolumn{2}{|c|}{$\begin{array}{c}\text { Alternative lengthening } \\
\text { of telomeres }(-)\end{array}$} \\
\hline & & & $\mathrm{N}(\%)$ & ATRX loss & $\mathrm{N}(\%)$ & ATRX loss \\
\hline Mesenchymal chondrosarcoma & 3 & $0 / 3(0 \%)$ & N/A & N/A & N/A & N/A \\
\hline Dermatofibrosarcoma protuberans & 10 & $0 / 10(0 \%)$ & $0 / 9(0 \%)$ & N/A & $9 / 9(100 \%)$ & $0 / 9(0 \%)$ \\
\hline Malignant solitary fibrous tumor & 7 & $0 / 7(0 \%)$ & $0 / 7(0 \%)$ & N/A & $7 / 7(100 \%)$ & $0 / 7(0 \%)$ \\
\hline Ewing sarcoma & 15 & $0 / 15(0 \%)$ & $0 / 10(0 \%)$ & N/A & $10 / 10(100 \%)$ & $0 / 10(0 \%)$ \\
\hline Alveolar rhabdomyosarcoma & 10 & $0 / 10(0 \%)$ & $0 / 8(0 \%)$ & N/A & $8 / 8(100 \%)$ & $0 / 8(0 \%)$ \\
\hline Synovial sarcoma & 18 & $0 / 18(0 \%)$ & $0 / 13(0 \%)$ & N/A & $13 / 13(100 \%)$ & $0 / 13(0 \%)$ \\
\hline Alveolar soft part sarcoma & 5 & $0 / 5(0 \%)$ & $0 / 4(0 \%)$ & N/A & $4 / 4(100 \%)$ & $0 / 4(0 \%)$ \\
\hline Extraskeletal myxoid chondrosarcoma & 3 & $0 / 3(0 \%)$ & $0 / 2(0 \%)$ & N/A & $2 / 2(100 \%)$ & $0 / 2(0 \%)$ \\
\hline Total & 71 & $0 / 71(0 \%)$ & $0 / 53(0 \%)$ & N/A & $53 / 53(100 \%)$ & $0 / 53(0 \%)$ \\
\hline \multicolumn{7}{|l|}{ Our previous studies ${ }^{\mathrm{a}}$} \\
\hline Myxoid liposarcoma & 20 & $0 / 20(0 \%)$ & $1 / 19(5 \%)$ & $0 / 1(0 \%)$ & 18/19 (95\%) & $0 / 18(0 \%)$ \\
\hline Epithelioid hemangioendothelioma & 11 & $1 / 11(9 \%)$ & $1 / 7(14 \%)$ & $1 / 1(100 \%)$ & $6 / 7(86 \%)$ & $0 / 6(0 \%)$ \\
\hline Total & 102 & $1 / 102(1 \%)$ & $2 / 79(3 \%)$ & $1 / 2(50 \%)$ & $77 / 79(97 \%)$ & $0 / 77(0 \%)$ \\
\hline \multicolumn{7}{|l|}{ Others } \\
\hline Gastrointestinal stromal tumor & 23 & $1 / 23(4 \%)$ & $1 / 16(6 \%)$ & $1 / 1(100 \%)$ & $15 / 16(94 \%)$ & $0 / 15(0 \%)$ \\
\hline Epithelioid sarcoma & 10 & $0 / 10(0 \%)$ & $0 / 6(0 \%)$ & N/A & $6 / 6(100 \%)$ & $0 / 6(0 \%)$ \\
\hline
\end{tabular}

Abbreviation: N/A, non-available.

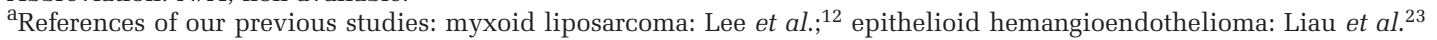

240 to $600 \mathrm{~s}$ for radiation-associated sarcomas, rhabdomyosarcomas, Ewing sarcomas, synovial sarcomas, and epithelioid sarcomas; and 120 to $240 \mathrm{~s}$ for dermatofibrosarcoma protuberans. Because most of the chondrosarcoma and osteosarcoma specimens had been subjected to a decalcification procedure, FISH was not performed for these tumor types.

The criteria used for interpretation of the FISH results were the same as previously defined. ${ }^{24}$ 


\section{Statistical Analysis}

Data analyses were conducted using SPSS Version 19 (IBM Corp., Armonk, NY, USA). Categorical variables were compared using the Pearson $\chi^{2}$ method or Fisher's exact test when appropriate. The statistical results were considered significant when $P<0.05$.

\section{Results}

\section{Loss of ATRX Expression in Sarcomas}

In total, 245 sarcomas were studied: 141 tumors with complex karyotypes, 71 gene fusion-associated sarcomas, 23 gastrointestinal stromal tumors, and 10 epithelioid sarcomas. Loss of ATRX expression was observed in 20 tumors (20/245, 8\%), including 19 sarcomas with complex cytogenetics $(19 / 141,13 \%)$, and one gastrointestinal stromal tumor $(1 / 23,4 \%)$. None of the fusion-associated sarcomas and epithelioid sarcomas was ATRX-deficient. All tumors showed preserved DAXX expression. Loss of ATRX expression was diffuse in essentially all tumor cells except one gastrointestinal stromal tumor, in which the loss of expression was focal (see below). Loss of ATRX expression was significantly more frequent in sarcomas with complex karyotypes than fusion-associated sarcomas $(P<0.001)$

\section{Alternative Lengthening of Telomeres in Sarcomas}

FISH results were available for 171 tumors: 96 sarcomas with complex cytogenetics, 53 sarcomas with fusion genes, 16 gastrointestinal stromal tumors, and 6 epithelioid sarcomas. In total, 49 tumors were alternative lengthening of telomeres positive $(49 / 171,29 \%)$, including 48 sarcomas with complex karyotypes (48/96, 50\%), and one gastrointestinal stromal tumor $(1 / 16,6 \%)$. None of the 53 sarcomas with fusion genes and the 6 epithelioid sarcomas was alternative lengthening of telomeres positive.

\section{Alternative Lengthening of Telomeres and Loss of ATRX Expression in Specific Sarcoma Types}

Approximately two-thirds (22/34, 65\%) of the undifferentiated pleomorphic sarcomas were alternative lengthening of telomeres positive (Figure 1a). Loss of ATRX was also common in undifferentiated pleomorphic sarcomas (12/35, 34\%). All ATRX-deficient undifferentiated pleomorphic sarcomas were alternative lengthening of telomeres positive.

Although most radiation-associated sarcomas can be classified as undifferentiated pleomorphic sarcoma, angiosarcoma, or leiomyosarcoma, ${ }^{25}$ 3 tumor types with frequent alternative lengthening of telomeres, only 3 of the 15 radiation-associated sarcomas were alternative lengthening of telomeres positive $(20 \%)$, and none was ATRX deficient.

Nineteen of 25 myxofibrosarcomas (76\%) were alternative lengthening of telomeres positive (Figure 1b). However, only 1 of the 27 myxofibrosarcomas was ATRX deficient (4\%); this ATRXdeficient tumor was alternative lengthening of telomeres positive. In our study, $2 / 2$ (100\%), $10 / 15(67 \%)$, and $7 / 8(88 \%)$ of the Fe'de'ration Nationale des Centres de Lutte Contre le Cancer Grade 1, 2, and 3 tumors, respectively, were alternative lengthening of telomeres positive, and no apparent correlation between the alternative lengthening of telomeres status and histological grade was noted.

Three of 14 malignant peripheral nerve sheath tumors were alternative lengthening of telomeres positive (21\%). One of the 3 alternative lengthening of telomeres-positive tumors showed loss of ATRX expression (Figure 1c). In this tumor, ATRX expression was lost in the malignant area but was retained in the neurofibromatous area.

One of the 9 embryonal rhabdomyosarcomas was ATRX deficient (11\%; Figure 2a). This ATRXdeficient tumor was the only tumor exhibiting anaplastic histological features and was also the only tumor with the alternative lengthening of telomeres phenotype.

Among the 23 gastrointestinal stromal tumors, 1 tumor exhibited focal loss of ATRX expression (4\%; Figure 2b). For this particular case, loss of ATRX expression was observed in a distinct nodule within the tumor, causing the tumor to have a tumor-in-tumor-like appearance. This nodule exhibited higher cellularity and mitotic activity compared with the background ATRX-proficient tumor cells. The tumor also exhibited medium-tolarge telomere signals in the hypercellular nodule, whereas telomere signals in the ATRX-proficient tumor cells outside of the nodule were small and uniform, similar to those in other alternative lengthening of telomeres-negative gastrointestinal stromal tumors.

Loss of ATRX expression was also seen in four conventional osteosarcomas (4/18, 22\%). All epithelioid sarcomas examined in this study showed preserved ATRX expression and were alternative lengthening of telomeres-negative (available in 6). ATRX expression was also retained in all 15 chondrosarcomas. Figure 2c shows the ATRX-deficient myxofibrosarcoma (C1-C2) and one ATRX-deficient osteosarcoma (C3-C4).

Figure 3 shows the representative histology, ATRX/DAXX immunohistochemistry, and telomere-specific FISH of the fusion-associated sarcomas (Figure 3a: malignant solitary fibrous tumor, Figure 3b: synovial sarcoma, Figure 3c: dermatofibrosarcoma protuberans). 

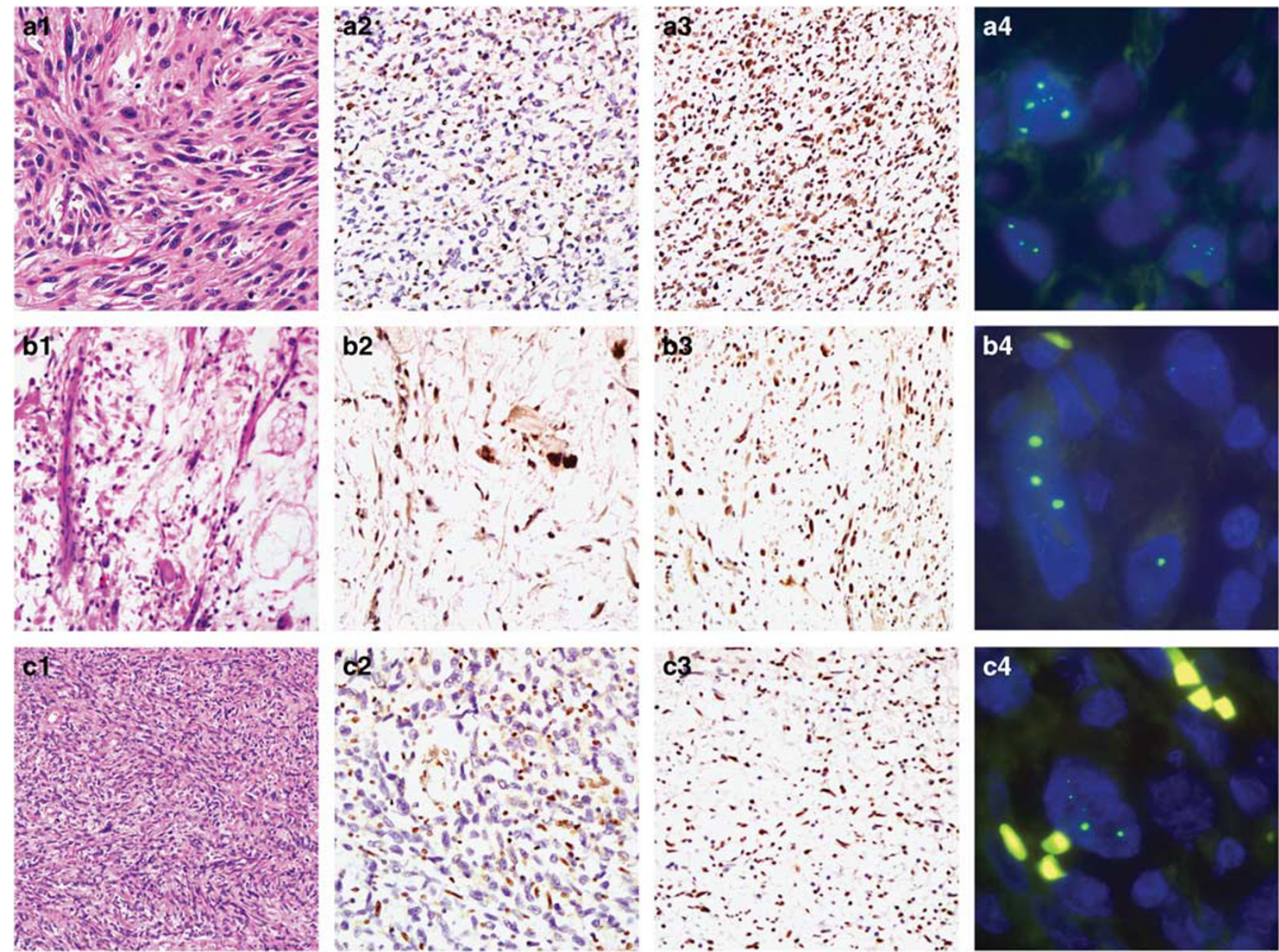

Figure 1 An undifferentiated pleomorphic sarcoma (a1) with loss of ATRX (a2) but not DAXX (a3) expression positive for alternative lengthening of telomeres phenotype (a4). A myxofibrosarcoma (b1) with preserved ATRX (b2) and DAXX (b3) expression positive for alternative lengthening of telomeres phenotype (b4). A malignant peripheral nerve sheath tumor (c1) with loss of ATRX expression in the sarcomatous area (c2) but not the neurofibromatous area (c3) positive for alternative lengthening of telomeres phenotype (c4). a1, b1, and c1: hematoxylin and eosin stain; a4, b4, and c4: telomere fluorescence in situ hybridization. Original magnification: a1-3, b1-3, and c1-3: $\times 200 ; \mathbf{a 4}$, b4, and $\mathbf{c 4 :} \times 1000$.

\section{Loss of ATRX Expression Was Highly Associated with Alternative Lengthening of Telomeres}

Tables 1 and 2 show the results of ATRX expression, alternative lengthening of telomeres, and their relationships (including the results of our previous studies). In combination with our previous studies and the present study, a total of 384 sarcomas with complex karyotypes were examined, 83 of which were ATRX deficient (22\%). Telomere-specific FISH revealed that $45 \%(138 / 308)$ were alternative lengthening of telomeres-positive, among which 55\% (76/138) were ATRX deficient. Loss of ATRX was significantly associated with alternative lengthening of telomeres $(P<0.001)$.

\section{Discussion}

In this study, we studied the frequency of alternative lengthening of telomeres and its correlation with loss of ATRX/DAXX expression in soft tissue sarcomas. After combining the results of this study and those from our previous works, we found that the frequency of this telomere-maintenance mechanism and its correlation with loss of ATRX expression were very similar among the leiomyosarcoma, undifferentiated pleomorphic sarcoma, and pleomorphic liposarcoma. All were characterized by alternative lengthening of telomeres in $>50 \%$ of the tumors and loss of ATRX expression was observed in approximately half of the alternative lengthening of telomeres-positive tumors. Similarly, studies have shown that $40-60 \%$ of osteosarcomas are alternative lengthening of telomeres positive, ${ }^{5,7}$ but ATRX gene mutation and loss of expression are detected in only $30 \%$ of pediatric osteosarcomas. ${ }^{20}$ Our results indicate that alternative lengthening of telomeres and loss of ATRX expression are common molecular characteristics of these pleomorphic sarcomas. It is possible that a significant subset of so-called undifferentiated pleomorphic sarcomas are 

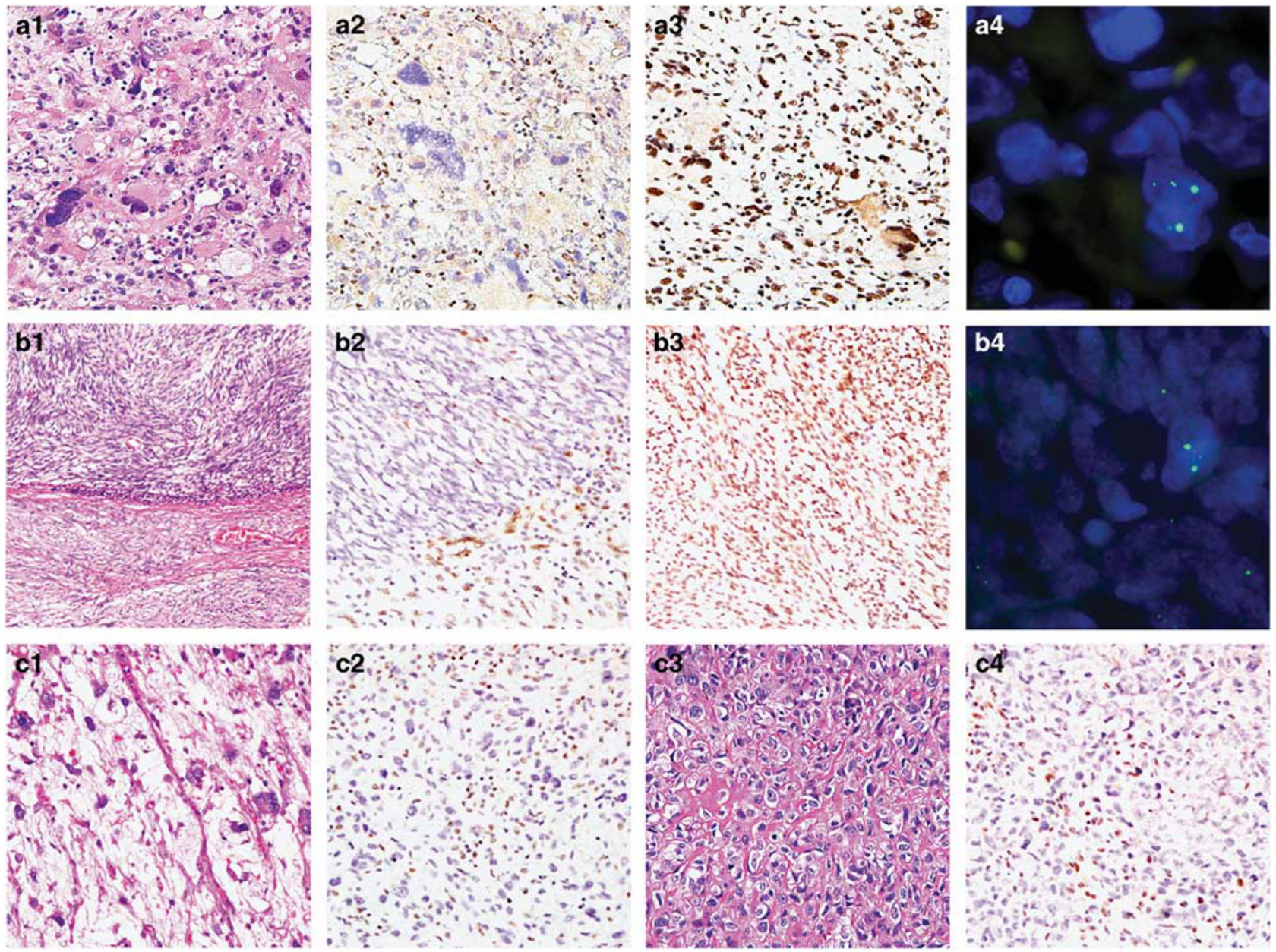

Figure 2 The anaplastic embryonal rhabdomyosarcoma (a1) with loss of ATRX (a2) but not DAXX (a3) expression positive for alternative lengthening of telomeres phenotype (a4). A hypercellular nodule in a gastrointestinal stromal tumor (b1) with loss of ATRX (b2) but not DAXX (b3) expression positive for alternative lengthening of telomeres phenotype (b4). The only myxofibrosarcoma (c1) deficient for ATRX (c2). An osteosarcoma (c3) deficient for ATRX (c4). a1, b1, c1, and c3: hematoxylin and eosin stain; a4 and b4: telomere fluorescence in situ hybridization. Original magnification: a1-3, b1-3, and c1-4: $\times 200$; $\mathbf{a 4}$ and b4: $\times 1000$.

other pleomorphic sarcomas such as dedifferentiated leiomyosarcomas, pleomorphic liposarcomas, or osteosarcomas in which the diagnostic features are no longer present or not identified. Alternatively, these pleomorphic sarcomas may have similar molecular features but exhibiting different lines and degrees of differentiation histologically.

In the present study, myxofibrosarcoma was frequently positive for alternative lengthening of telomeres. Histologically, several other sarcoma types, such as undifferentiated pleomorphic sarcoma, pleomorphic liposarcoma, and dedifferentiated liposarcoma, may have focally a myxofibrosarcoma-like appearance. However, the vast majority of myxofibrosarcomas were ATRX-proficient, suggesting that myxofibrosarcoma has a unique molecular pathogenesis for the alternative lengthening of telomeres phenotype. Increased histological grades and cytogenetic aberrations have been observed in the recurrence of myxofibrosarcoma. ${ }^{26}$ However, the prevalence of alternative lengthening of telomeres was similar among the Grade 1 to 3 tumors in our study, indicating that alternative lengthening of telomeres is a common feature across all tumor grades. The minimal amount of myxoid stroma required for the diagnosis of myxofibrosarcoma is debatable and some authors argue that tumors possess $<50 \%$ of myxoid stroma should be classified as undifferentiated pleomorphic sarcomas. This ATRX-deficient myxofibrosarcoma had a multinodular appearance and two-thirds of the tumor was myxoid. In the myxoid area, there were long and branching thin walled vascular channels. We believe that this tumor best fits the diagnosis of myxofibrosarcoma but additional studies are necessary to determine whether such ATRX-deficient myxofibrosarcomalike tumors are better classified as undifferentiated pleomorphic sarcomas.

Most radiation-associated sarcomas exhibit an undifferentiated morphology. However, only $20 \%$ of radiation-associated sarcomas were alternative 

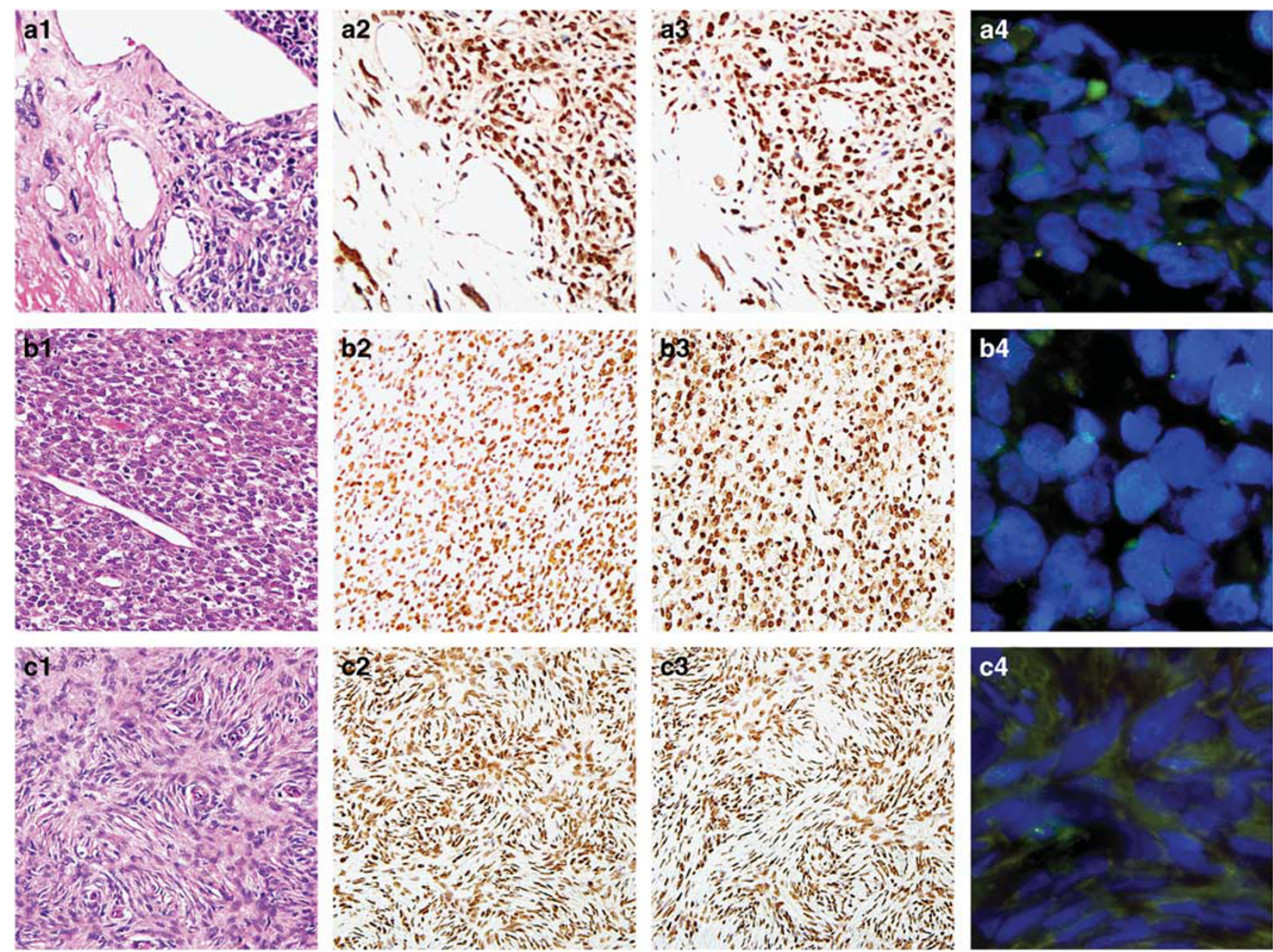

Figure 3 A malignant solitary fibrous tumor (a1) with preserved ATRX (a2) and DAXX (a3) expression negative for alternative lengthening of telomeres phenotype (a4). A poorly differentiated synovial sarcoma (b1) with preserved ATRX (b2) and DAXX (b3) expression negative for alternative lengthening of telomeres phenotype (b4). A dermatofibrosarcoma protuberans (c1) with preserved ATRX (c2) and DAXX (c3) expression negative for alternative lengthening of telomeres phenotype (c4). a1, b1, and c1: hematoxylin and eosin stain; a4, b4, and c4: telomere fluorescence in situ hybridization. Original magnification: a1-a3, b1-b3, and $\mathbf{c 1 - c 3 : ~} \times 200$; a4, b4, and c4: $\times 1000$.

lengthening of telomeres positive, which was significantly lower than that of undifferentiated pleomorphic sarcomas $(P=0.005)$. Moreover, none of the radiation-associated sarcomas was ATRX deficient. These observations indicate that, although morphologically similar, the pathogenesis of radiation-associated sarcoma differs from that of de novo undifferentiated pleomorphic sarcoma. Highdose radiation induces multiple double-strand breaks in chromosomes and breakage-fusion-bridge cycles, resulting in a gain of oncogenes, loss of tumor suppressor genes, and activation of DNA damage responses. ${ }^{27}$ Telomerase expression can be activated during this process. ${ }^{28}$ Therefore, ATRX loss and alternative lengthening of telomeres might not be needed for immortalization of radiation-associated tumors.

Telomerase expression is the main telomeremaintenance mechanism in high-risk gastrointestinal stromal tumors. ${ }^{29}$ Although the tumor cells in gastrointestinal stromal tumors are typically relatively uniform, exceptional cases of 'dedifferentiated' tumors with anaplastic features, and heterologous rhabdomyosarcomatous differentiation following tyrosine kinase inhibitor treatment have been reported. ${ }^{30,31}$ Recently, a KRAS-mutated KIT/PDGFRA-wild type gastrointestinal stromal tumor was found to have ATRX mutations. ${ }^{32}$ This tumor also exhibited anaplastic histological features and behaved aggressively. We speculate that loss of ATRX expression and/or acquisition of alternative lengthening of telomeres might be associated with dedifferentiation and/or transdifferentiation of gastrointestinal stromal tumors, and convey a poor prognosis.

Only a minority of malignant peripheral nerve sheath tumors, gastrointestinal stromal tumors, and embryonal rhabdomyosarcomas were alternative lengthening of telomeres positive or ATRX deficient (or both). Interestingly, these cases hinted the 
possibility that alternative lengthening of telomeres and loss of ATRX might be involved in the dedifferentiation or anaplastic change. In the ATRX-deficient malignant peripheral nerve sheath tumor, loss of ATRX expression was restricted to the malignant area. In the ATRX-deficient gastrointestinal stromal tumor, loss of expression was restricted to the hypercellular nodule. In embryonal rhabdomyosarcomas, the only ATRX-deficient case was also the only tumor exhibiting anaplastic histological features. Previous studies have shown that in contrast to non-anaplastic embryonal rhabdomyosarcomas, anaplastic tumors were characterized by more complex cytogenetic derangements with gene amplifications. ${ }^{33,34}$ In keeping with this hypothesis, in our previous study, none of the well-differentiated liposarcomas was alternative lengthening of telomeres positive, but $30 \%(14 / 46)$ of the dedifferentiated liposarcomas were alternative lengthening of telomeres positive. ${ }^{12}$

Except rare cases of myxoid liposarcoma and epithelioid hemangioendothelioma, ${ }^{12,23}$ none of the gene fusion-associated sarcomas in our studies was ATRX-deficient or alternative lengthening of telomeres positive, indicating that alternative lengthening of telomeres is not an important telomeremaintenance mechanism for fusion-associated sarcomas. Interestingly, TERT promoter mutation, a crucial mechanism of telomerase activation, is also uncommon in fusion-associated sarcomas. ${ }^{35} \mathrm{~A}$ possible explanation for these observations is that fusion-associated sarcomas typically occur in young patients and might be more likely to be derived from stem cells with intrinsic telomerase activity. Another possibility is that the oncogenic fusion proteins might activate telomerase expression, such as the EWSR1/ETS fusions in Ewing sarcomas. ${ }^{36}$ One noteworthy exception is myxoid/round liposarcomas, which have TERT promoter mutation in approximately $70-80 \%$ of cases. ${ }^{35}$ A previous study also showed that telomerase expression was the main telomere-maintenance mechanism in myxoid/ round cell liposarcomas. ${ }^{11}$ Patients with myxoid/ round liposarcoma are older than those with most other types of fusion-associated sarcomas. It is also possible that the fusion proteins in myxoid liposarcoma are insufficient for activating telomerase expression.

Epithelioid sarcoma is characterized by genetic alterations involving the tumor suppressor gene $S M A R C B 1 .^{37}$ In contrast to a previous study which showed two of six epithelioid sarcomas were alternative lengthening of telomeres positive, ${ }^{24}$ all tumors in our series were alternative lengthening of telomeres-negative and ATRX-proficient. Our results suggest that epithelioid sarcomas might be more similar to fusion-associated sarcomas and gastrointestinal stromal tumors, in which a dominant genetic change drives the tumor formation, and alternative lengthening of telomeres does not play important roles in the pathogenesis.
A potential caveat of our study is that some ATRX-mutated sarcomas may exhibit preserved ATRX immunostaining. We used antibodies recognizing the C-terminal of ATRX and DAXX proteins, which have been previously shown to be highly correlated with alternative lengthening of telomeres in pancreatic neuroendocrine tumors. ${ }^{13}$ However, theoretically, some point mutations might affect protein function without abolishing protein expression. Therefore, the immunostaining assay might underestimate the frequency of dysfunctional ATRX protein. Future studies using sequencing analysis might reveal the frequency of mutation in these genes.

In summary, we have screened a large variety of sarcomas for alternative lengthening of telomeres status and ATRX/DAXX expression. Combining the results of this study with those of our previous studies, we have shown that alternative lengthening of telomeres is an important telomere-maintenance mechanism in sarcomas with complex cytogenetics and is frequently associated with loss of ATRX expression. However, its correlation with loss of ATRX expression differ among the sarcoma types and, for some tumors, particularly the myxofibrosarcomas, alternative lengthening of telomeres appears to be attributable to mechanisms other than loss of ATRX in most cases. Alternative lengthening of telomeres and loss of ATRX are rare in simple karyotype sarcomas, but they might have roles in high-grade transformation and/or dedifferentiation. Recently, ataxia telangiectasia and Rad3-related kinase (ATR) was shown to play crucial roles in homologous recombination and alternative lengthening of telomeres, and inhibition of this kinase caused apoptosis of alternative lengthening of telomeres-positive cancer cells. ${ }^{38}$ Identification of alternative lengthening of telomeres and loss of ATRX in sarcomas may provide new opportunities to treat these aggressive neoplasms.

\section{Acknowledgments}

This work is supported by grants MOST 103-2320B-002-021 to J-Y Liau, and MOST 104-2314-B-002029 and NTUH 104-P12 to C-Y Yang.

\section{Disclosure/conflict of interest}

The authors declare no conflict of interest.

\section{References}

1 Antonescu CR. The role of genetic testing in soft tissue sarcoma. Histopathology 2006;48:13-21.

2 Gunes C, Rudolph KL. The role of telomeres in stem cells and cancer. Cell 2013;152:390-393. 
3 Cesare AJ, Reddel RR. Alternative lengthening of telomeres: models, mechanisms and implications. Nat Rev Genet 2010;11:319-330.

4 Royle NJ, Foxon J, Jeyapalan JN et al. Telomere length maintenance-an ALTernative mechanism. Cytogenet Genome Res 2008;122:281-291.

5 Henson JD, Hannay JA, McCarthy SW et al. A robust assay for alternative lengthening of telomeres in tumors shows the significance of alternative lengthening of telomeres in sarcomas and astrocytomas. Clin Cancer Res 2005;11:217-225.

6 Liau JY, Tsai JH, Jeng YM et al. Leiomyosarcoma with alternative lengthening of telomeres is associated with aggressive histologic features, loss of ATRX expression, and poor clinical outcome. Am J Surg Pathol 2015;39: 236-244.

7 Ulaner GA, Hoffman AR, Otero J et al. Divergent patterns of telomere maintenance mechanisms among human sarcomas: sharply contrasting prevalence of the alternative lengthening of telomeres mechanism in Ewing's sarcomas and osteosarcomas. Genes Chromosomes Cancer 2004;41:155-162.

8 Montgomery E, Argani P, Hicks JL et al. Telomere lengths of translocation-associated and nontranslocationassociated sarcomas differ dramatically. Am J Pathol 2004;164:1523-1529.

9 Venturini L, Motta R, Gronchi A et al. Prognostic relevance of ALT-associated markers in liposarcoma: a comparative analysis. BMC Cancer 2010;10:254.

10 Ulaner GA, Huang HY, Otero J et al. Absence of a telomere maintenance mechanism as a favorable prognostic factor in patients with osteosarcoma. Cancer Res 2003;63:1759-1763.

11 Costa A, Daidone MG, Daprai L et al. Telomere maintenance mechanisms in liposarcomas: association with histologic subtypes and disease progression. Cancer Res 2006;66:8918-8924.

12 Lee JC, Jeng YM, Liau JY et al. Alternative lengthening of telomeres and loss of ATRX are frequent events in pleomorphic and dedifferentiated liposarcomas. Mod Pathol 2015;28:1064-1073.

13 Heaphy CM, de Wilde RF, Jiao Y et al. Altered telomeres in tumors with ATRX and DAXX mutations. Science 2011;333:425.

14 Drane P, Ouararhni K, Depaux A et al. The deathassociated protein DAXX is a novel histone chaperone involved in the replication-independent deposition of H3.3. Genes Dev 2010;24:1253-1265.

15 Goldberg AD, Banaszynski LA, Noh KM et al. Distinct factors control histone variant H3.3 localization at specific genomic regions. Cell 2010;140:678-691.

16 Wong LH, McGhie JD, Sim M et al. ATRX interacts with H3.3 in maintaining telomere structural integrity in pluripotent embryonic stem cells. Genome Res 2010;20:351-360.

17 Liu XY, Gerges N, Korshunov A et al. Frequent ATRX mutations and loss of expression in adult diffuse astrocytic tumors carrying IDH1/IDH2 and TP53 mutations. Acta Neuropathol 2012;124:615-625.

18 Lovejoy CA, Li W, Reisenweber S et al. Loss of ATRX, genome instability, and an altered DNA damage response are hallmarks of the alternative lengthening of telomeres pathway. PLoS Genet 2012;8:e1002772.

19 Fishbein L, Khare S, Wubbenhorst B et al. Whole-exome sequencing identifies somatic ATRX mutations in pheochromocytomas and paragangliomas. Nat Commun 2015;6:6140.
20 Chen X, Bahrami A, Pappo A et al. Recurrent somatic structural variations contribute to tumorigenesis in pediatric osteosarcoma. Cell Rep 2014;7:104-112.

21 Pinto EM, Chen X, Easton J et al. Genomic landscape of paediatric adrenocortical tumours. Nat Commun 2015;6:6302.

22 Gibbons RJ, Picketts DJ, Villard L et al. Mutations in a putative global transcriptional regulator cause X-linked mental retardation with alpha-thalassemia (ATR-X syndrome). Cell 1995;80:837-845.

23 Liau JY, Tsai JH, Yang CY et al. Alternative lengthening of telomeres phenotype in malignant vascular tumors is highly associated with loss of ATRX expression and is frequently observed in hepatic angiosarcomas. Hum Pathol 2015;46:1360-1366.

24 Heaphy CM, Subhawong AP, Hong SM et al. Prevalence of the alternative lengthening of telomeres telomere maintenance mechanism in human cancer subtypes. Am J Pathol 2011;179:1608-1615.

25 Gladdy RA, Qin LX, Moraco N et al. Do radiationassociated soft tissue sarcomas have the same prognosis as sporadic soft tissue sarcomas? J Clin Oncol 2010;28:2064-2069.

26 Willems SM, Debiec-Rychter M, Szuhai K et al. Local recurrence of myxofibrosarcoma is associated with increase in tumour grade and cytogenetic aberrations, suggesting a multistep tumour progression model. Mod Pathol 2006;19:407-416.

27 Lefevre SH, Vogt N, Dutrillaux AM et al. Genome instability in secondary solid tumors developing after radiotherapy of bilateral retinoblastoma. Oncogene 2001;20:8092-8099.

28 Ram R, Uziel O, Eldan O et al. Ionizing radiation upregulates telomerase activity in cancer cell lines by post-translational mechanism via ras/phosphatidylinositol 3-kinase/Akt pathway. Clin Cancer Res 2009;15: 914-923.

29 Sakurai S, Fukayama M, Kaizaki Y et al. Telomerase activity in gastrointestinal stromal tumors. Cancer 1998;83:2060-2066.

30 Antonescu CR, Romeo S, Zhang L et al. Dedifferentiation in gastrointestinal stromal tumor to an anaplastic KIT-negative phenotype: a diagnostic pitfall: morphologic and molecular characterization of 8 cases occurring either de novo or after imatinib therapy. Am J Surg Pathol 2013;37:385-392.

31 Liegl B, Hornick JL, Antonescu CR et al. Rhabdomyosarcomatous differentiation in gastrointestinal stromal tumors after tyrosine kinase inhibitor therapy: a novel form of tumor progression. Am J Surg Pathol 2009;33:218-226.

32 Hechtman JF, Zehir A, Mitchell T et al. Novel oncogene and tumor suppressor mutations in KIT and PDGFRA wild type gastrointestinal stromal tumors revealed by next generation sequencing. Genes Chromosomes Cancer 2015;54:177-184.

33 Bridge JA, Liu J, Qualman SJ et al. Genomic gains and losses are similar in genetic and histologic subsets of rhabdomyosarcoma, whereas amplification predominates in embryonal with anaplasia and alveolar subtypes. Genes Chromosomes Cancer 2002;33: 310-321.

34 Qualman S, Lynch J, Bridge J et al. Prevalence and clinical impact of anaplasia in childhood rhabdomyosarcoma: a report from the Soft Tissue Sarcoma Committee of the Children's Oncology Group. Cancer 2008;113:3242-3247. 
35 Koelsche C, Renner M, Hartmann W et al. TERT promoter hotspot mutations are recurrent in myxoid liposarcomas but rare in other soft tissue sarcoma entities. J Exp Clin Cancer Res 2014;33:33.

36 Takahashi A, Higashino F, Aoyagi $\mathrm{M}$ et al. EWS/ETS fusions activate telomerase in Ewing's tumors. Cancer Res 2003;63:8338-8344.
37 Modena P, Lualdi E, Facchinetti F et al. SMARCB1/ INI1 tumor suppressor gene is frequently inactivated in epithelioid sarcomas. Cancer Res 2005;65: 4012-4019.

38 Flynn RL, Cox KE, Jeitany $\mathrm{M}$ et al. Alternative lengthening of telomeres renders cancer cells hypersensitive to ATR inhibitors. Science 2015;347:273-277. 